\title{
LAGUERRE-GRAM REDUCED ORDER MODELING APPLIED TO VLSI CIRCUIT INTERCONNECTS
}

\author{
M. Telescu, P. Brehonnet, N. Tanguy, P. Vilbé, L.C. Calvez \\ LEST UMR CNRS n6165 \\ 6, avenue Le Gorgeu, CS 63837, 29238 Brest Cedex 3, France \\ E-mail: Mihai.Telescu@univ-brest.fr
}

\begin{abstract}
Reduced order modeling has become a vital tool for decreasing computational cost in time domain simulations. In this paper we present a Laguerre-Gram model-order reduction technique applied to admittance matrices of circuit interconnect lines. We show reduction results for a single line and for a system of two coupled lines but also an iterative solution for obtaining better low order approximations of delayed signals.
\end{abstract}

\section{INTRODUCTION}

The current tendency of the VLSI industry is to decrease component size while increasing working frequencies. Simple interconnects in these ever smaller, ever faster circuits may have effects such as delay, noise, reflections or cross talk. It often proves difficult and time consuming for a circuit designer to take these effects into consideration during simulation. Tools have therefore been developed to enable the approximation of full interconnect models by simpler, more versatile ones. The method we propose in this paper benefits from recent research on Laguerre representations [2],[6].

\section{REDUCTION METHOD}

Our aim is to approximate infinite or very high order functions $\hat{f}(s)$, representing admittances, impedances or system transfer functions, with rational models of an $r$ order as low as possible. Finding an appropriate rational model, especially its denominator, is not a linear problem. The method we propose circumvents this difficulty. To start with, any function $\hat{f}(s)$ representing the Laplace transform of $f(t) \in L^{2}\left(R^{+}\right)$can be decomposed in a Laguerre series

$$
\hat{f}(s)=\sum_{n=0}^{\infty} f_{n} \hat{\phi}_{n}(s)
$$

We then build an array of transfer functions $\Omega_{r}=\left\{\hat{f}_{0}, \hat{f}_{1}, \ldots, \hat{f}_{r}\right\}$ obtained by successive applications of the operator denoted as $\Lambda$ and defined by:

$$
\begin{aligned}
& \hat{f}_{0}(s)=\hat{f}(s), \\
& \hat{f}_{i+1}(s) \hat{=} \Lambda \hat{f}_{i}(s)=\left[\hat{f}_{i}(s)-(2 \alpha) /(s+\alpha) f_{i}(\alpha)\right](s+\alpha) /(s-\alpha) \\
& i=0, \ldots, r-1
\end{aligned}
$$

An interesting property of this operator is that it preserves the poles and natural frequencies of $\hat{f}(s)$. Denoting as $\left\{f_{i, n}\right\}_{n \geq 0}$ the Laguerre coefficients of the $\hat{f}_{i}(s)$ function, the Laguerre spectrum (coefficient array) of $\hat{f}_{i+1}(s)$ is obtained in a quite obvious manner from that of $\hat{f}_{i}(s)$ and so on

$$
f_{i+1, n}=f_{i, n+1}=\ldots=f_{0, i+n+1}
$$

for any $n \geq 0, i \geq 0$. This property makes the algorithm advantageous from a computational time point of view. It can be shown that the approximation of $\hat{f}_{r}(s)$ as linear combination of the other $r$ - 1 functions belonging to $\Omega_{r}$ enables the construction of a reduced rational model of $\hat{f}(s)$. Let $\vec{a}=\left[a_{0}, a_{1}, \ldots, a_{r-1}\right]^{T}$ denote the coefficients of this linear combination. The best $r$ order $\vec{a}=\left[a_{0}, a_{1}, \ldots, a_{r-1}\right]^{T}$ vector which minimizes the mean square error (MSE) is the solution to the system of equations given by

$\Psi \vec{a}=-\vec{b}$

where $\Psi$ denotes the Gram matrix of inner products $\psi_{i, j}=\left\langle\hat{f}_{i}, \hat{f}_{j}\right\rangle$ for $i, j=0,1, \ldots, r-1$ and $\vec{b}$ the vector $\left[\psi_{0, r}, \psi_{1, r}, \ldots, \psi_{r-1, r}\right]^{T}$ and ${ }^{T}$ the transpose. A reduced rational model $\hat{\tilde{f}}(s)$ is then easily constructed from $\vec{a}$ [2]. Preserving this model's denominator, it is a classical linear problem to find a numerator optimized in the sense of minimizing $\|f-\tilde{f}\|^{2}$. Moreover, the method has the remarkable property of preserving system stability. 
It is also worth noting that inner products can be expressed exclusively from the Laguerre spectrum of $\hat{f}(s)$

$\psi_{i, j}=\sum_{n=0}^{\infty} f_{n+i} f_{n+j}$

They are thus replaced by sums, in practice finite, and are therefore very easy to calculate.

\section{REDUCTION METHOD APPLIED TO INTERCONNECTS}

\subsection{Reduced admittance models}

Rather than consider the chain matrix or the voltage to voltage transfer function as done in previous work [4], we chose to describe the interconnect by its admittance matrix:

$$
\left(\begin{array}{c}
i_{\text {input }} \\
i_{\text {output }}
\end{array}\right)=\left(\begin{array}{ll}
Y_{11} & Y_{12} \\
Y_{21} & Y_{22}
\end{array}\right)\left(\begin{array}{c}
v_{\text {input }} \\
v_{\text {output }}
\end{array}\right)
$$

The first advantage of such a representation is that this matrix is persymmetrical, thus we obtain a complete description of the circuit with only two matrix terms. But what is even more important is that it greatly facilitates future work on passivity enforcement. For a system of $n$ coupled lines we have an admittance matrix of irrational (infinite order) functions and is given by

$$
\begin{aligned}
& Y_{11}=Y_{22}=(Y Z)^{1 / 2}\left[Z \cdot \sinh (Y Z)^{1 / 2}\right]^{-1} \cdot \cosh (Y Z)^{1 / 2} \\
& Y_{12}=Y_{21}=-(Y Z)^{1 / 2}\left[Z \cdot \sinh (Y Z)^{1 / 2}\right]^{-1}
\end{aligned}
$$

where $Z$ and $Y$ are square matrices, their size given by the number of coupled lines. They represent the series and, respectively, parallel contributions of line parameters. In this paper we will only focus on the trans-admittance term $Y_{12}$ which contains a pure delay and is therefore much harder to approximate. The reduction algorithm used for $Y_{11}$ is identical and comparative results are available in [8] and [9]. Physically, $\mathrm{Y}_{12}$ is the expression of a short circuit output current as a function of the input voltage. In this paper we will presume that the input voltage is a $1 \mathrm{~V}$ step excitation applied at time 0 .

\subsection{Reduction results for a single line}

We consider a $2 \mathrm{~mm}$ interconnect characterized by the following line parameters: $\mathrm{R}=72852 \Omega, \mathrm{C}=100 \mathrm{pF}$, $\mathrm{L}=1.08 \mu \mathrm{H}$. The step response for $\mathrm{Y}_{12}$ is shown in figure 2 as well as the response of an order 30 reduced model. The overall MSE in this case is of $1.6 \%$.

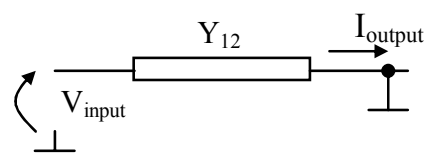

Figure 1. Single line configuration

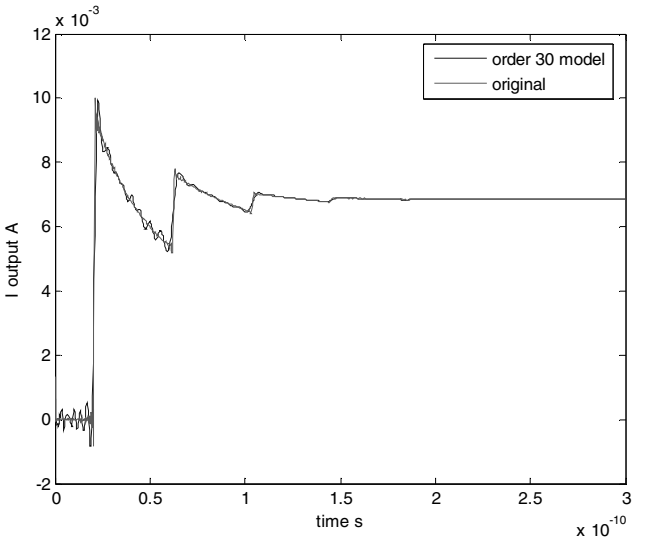

Figure 2. $\mathrm{Y}_{12}$ step response for a single line and its order 30 reduced model

On a more subjective level, it worth noting that both the delay introduced by the interconnect and the reflections remain visible on the reduced model.

\subsection{Reduction results for two coupled lines}

At this point we consider two coupled interconnect lines, identical to the one described in paragraph 3.2. $\mathrm{M}=0.804$ $\mu \mathrm{H}$ and $\mathrm{Cc}=59.3 \mathrm{pF}$ are, respectively, the mutual inductance and the capacitance modeling the coupling effect. The configuration is shown in figure 3 .

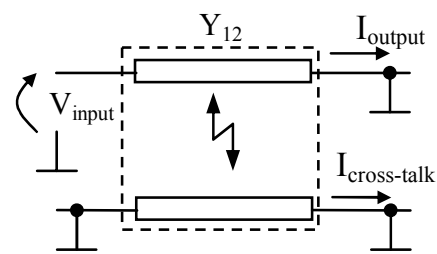

Figure 3. Two coupled lines configuration

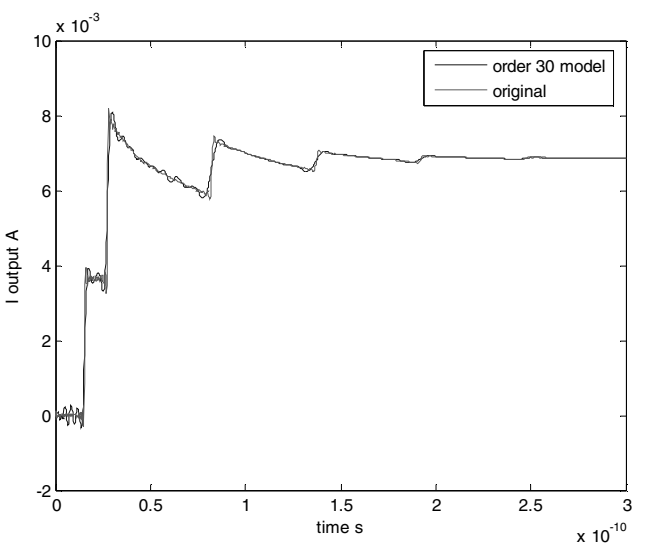

Figure $4 \mathrm{Y}_{12}$ direct step response for two coupled lines and its order 30 model 


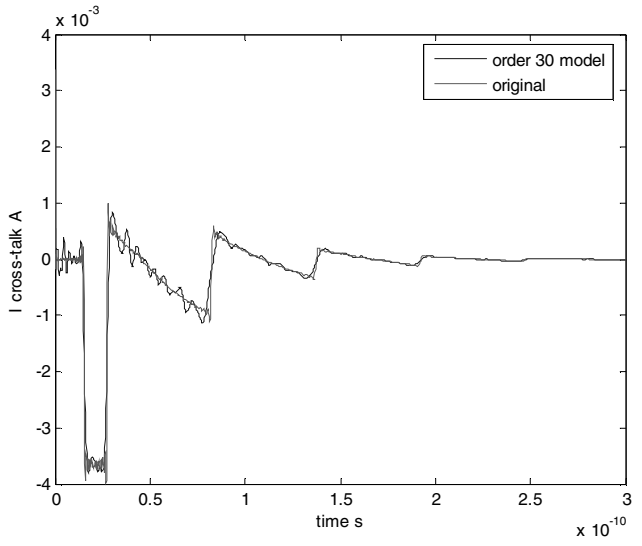

Figure $5 \mathrm{Y}_{12}$ cross-talk step response for two coupled lines and its order 30 model

By comparing figures 2 and 4 we can see the coupling effects, especially on the delay. The order 30 reduced order model has an error of $0.61 \%$ In figure 5 we study the cross-talk current injected in the second line. We provide an order 30 reduced order model with an error of $4.1 \%$. The difference in terms of MSE between the models presented in figures 4 and 5 is explained by the presence of a second almost vertical front in cross-talk current which makes reduction more difficult in this case.

\subsection{Iterative model reduction}

Reduction methods have some difficulties dealing with a pure delay, especially when relatively small orders are desired.

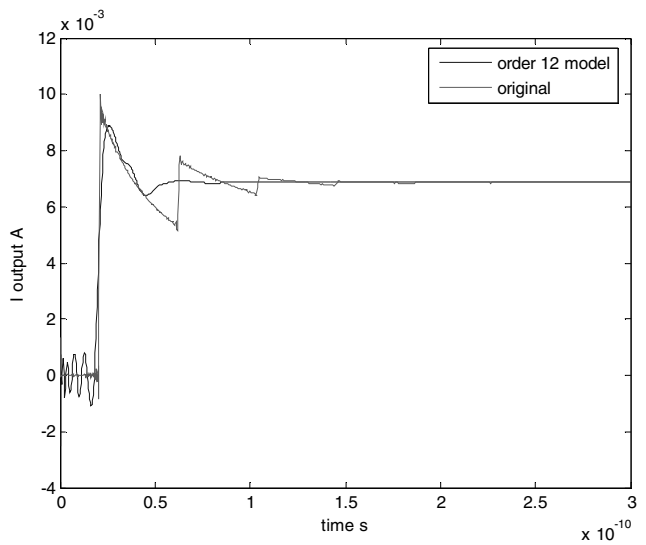

Figure 6. $Y_{12}$ step response and the order 12 model obtained by direct reduction

Previous works have suggested an improvement [4]. The original function is decomposed into several signals, corresponding to different propagation modes, delay is extracted on each of these signals which are then reduced sepparatly and a global model is finally reconstructed. But passivity enforcement becomes much more difficult in this case and the approach is not desirable if we are to find an equivalent circuit for the reduced model. A solution allowing us to obtain small order models that include delays relies upon an unexpected property of the $\Lambda$ operator. The iterative application of this operator on a function, with a gradual decrease in order, may radically improve its efficiency, especially for signals containing delays. For example: rather than search for an order 12 reduced model of the original irrational function, we will search for an order 24 and then approximate this order 24 by an order 12 [9]. The improvement in terms of MSE can be important without drastically increasing computation time. The order 12 approximation shown in figure 6 is obtained by direct reduction and has a $6.3 \%$ error.

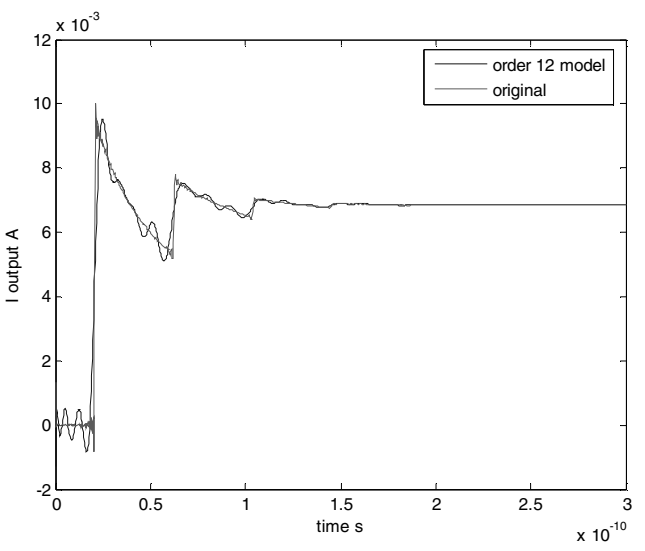

Figure 7. Y12 step response and an order 12 model obtained after 4 iterations

We then perform an iterative reduction in 4 steps (figure 7 ) with an initialization order of 24 . The error decreases at $3.9 \%$. The improvement is in fact quite visible when comparing the degree to which the two models preserve the effects of reflection. We also note that for longer interconnect lines the difference between iterative and direct reduction is even more striking [8]. Such cases are, however, less common in VLSI applications.

\section{CONCLUSION}

The model-order reduction technique presented here is essentially based on the use of a simple linear operator applied to a Laguerre representation. In the context of VLSI interconnections modeling we chose to work with the admittance matrix and we show that an iterative approach can decrease MSE for delayed signals. The perspectives of our work would be to find a simple equivalent circuit for the reduced model and to extend our method to more complex structures. We may also benefit from the fact that our method allows the frequency dispersion of R,L,C,G parameters to be taken into consideration while traditional segmentation methods do not.

\section{ACKNOWLEDGEMENTS}

The authors would like to acknowledge Brittany Region's financial support. 


\section{REFERENCES}

[1] W.T. Weeks, Numerical inversion of Laplace transforms using Laguerre functions, J.ACM, vol 13, pp 419-426, 1966.

[2] A. Amghayrir, N. Tanguy, P. Bréhonnet, P. Vilbé, L.C.Calvez, Laguerre-Gram Reduced-Order Modeling, IEEE Transactions on Automatic Control, accepted for publication.

[3] C. Nouët, Réduction de l'ordre des systèmes continus linéaires via un processus d'orthogonalisation et un algorithme de Gauss-Newton, Thèse de Doctorat, Université de Bretagne Occidentale, Brest, 1994

[4] A. Amghayrir, P. Brehonnet, N. Tanguy, P. Vilbé, L.C. Calvez, F. Huret, A Frequency Domain Approach for Efficient Model Reduction of Mixed VLSI Circuits, 8th IEEE Workshop on Signal Propagation On Interconnects, Heidelberg, Germany, 8-11 May 2004.

[5] M. Sevellec, Réduction de l'ordre des systèmes à fonctions de transfert rationnel, Thèse de Doctorat, Université de Bretagne Occidentale, Brest, 1993

[6] N. Tanguy, P. Brehonnet, P. Vilbé, L.C Calvez, Gram matrix of a Laguerre model: application to model reduction of irrational transfer function, Signal Processing, vol. 85, n 3, pp. 651-655, March 2005

[7] P. Bréhonnet , T. Le Gougec, N. Tanguy, P.M. Martin, D. Descacht, P. Vilbé, L.C. Calvez, F. Huret, A efficient model-order reduction for a complete fullwave electromagnetic analysis of SOC-AMS, 7th IEEE Workshop on Signal Propagation On Interconnects, Siena, Italy, 11-14 May 2003

[8] M Telescu, P. Brehonnet, N. Tanguy, P. Vilbé, L.C. Calvez, F. Huret, Model-Order Reduction of VLSI Circuit Interconnects via a Laguerre representation, 9th IEEE Workshop on Signal Propagation On Interconnects, Heidelberg, Germany, 10-13 May 2005.

[9] M Telescu, P. Brehonnet, N. Tanguy, P. Vilbé, L.C. Calvez, Réduction de modèle des interconnexions de circuits VLSI via une représentation de Laguerre, 14èmes Journées Nationales Microondes, Nantes, France, May 2005 